\title{
Study on the Status and Development Prospect of the Nano-Concrete
}

\author{
Wei $\mathrm{Li}^{1,2,3, \text { a }}$, Yanming Zhao ${ }^{1, \mathrm{~b}}$, Xiaochu Wang ${ }^{1,2, \mathrm{c}}$, Xi Yang ${ }^{1, \mathrm{~d}}$ \\ ${ }^{1}$ Shenyang University, Liaoning, Shenyang, 110044, China \\ ${ }^{2}$ The Key Laboratory of Geoenvironmental Engineering, Liaoning, Shenyang, 110044, China \\ ${ }^{3}$ Pollution Environmental Governance and Key Laboratory of Regional Ecological Security, \\ Liaoning, Shenyang, 110044, China \\ asydxIw@163.com(corresponding author), b975134774@qq.com, \\ cwangxiaochu@126.com, ${ }^{\mathrm{d}} 787334571 @ q q . c o m$
}

\begin{abstract}
Keywords: Nano-Concrete , Mechanical Properties, Durability
Abstract: Since the advent of nano-materials, its unique mechanical properties and chemical properties has been the concern of many experts and scholars. Describes the application of research results in recent years and nano-materials in the construction industry. Adding nano-materials in ordinary concrete can improve the performance of concrete including strength, toughness, durability and other properties. In recent years, with the increase in the number of nano-materials researchers, nano-materials we use in the construction industry also have a better understanding, but not yet fully grasp its mechanism of action. So we need more in-depth research and exploration.
\end{abstract}

\section{Introduction}

Nano materials started in twentieth Century 30 in the 1980s. When Japan study it for its military purposes "heavy smoke test", the first nano-materials in the world superfine lead powder has been made by vacuum evaporation method successfully. But its performance is not stable because of the technical condition of the time limit. Nano-materials have really attracted many researchers' interest is in twentieth Century 80's. America held the first session of the international nano technology conference in 1990 July, and confirmed the nano materials science is a new branch of material science officially. Because of its outstanding performance in the field of architecture, medicine, physics, chemistry, machinery, materials and so on, it attracts many researchers' gaze.

\section{The Characteristics of Nano-materials}

The nano-meter is a unit of length, one nano-meter is one-billionth of a meter. Composed with fine particles of magnitude, nano-materials are nano-scale in size between $1 \mathrm{~nm}-100 \mathrm{~nm}$. Because of its very small particles, it has its own unique character.

Nano-materials exist volume effect. The so-called volume effect refers to the size of the particles. When nano-materials and de Broglie wave looks similar or smaller, its periodic boundary conditions will be destroyed, leading to its physical properties, chemical properties, etc. The performance relative to conventional materials have great changes.

Nano-materials have size effect. The so-called size effect is that when the particle size reduced to a certain extent, the electronic level spacing split occurred. Fluctuations in nano-particles electronic nano-materials gives it new features, such as photo catalysis and so on.

Nano-materials having a surface effect. The surface effect is that as the particle size is reduced, the number of surface atoms have a sharp increase and the surface area of nano-particles is also increasing rapidly. Due to the lack of other atoms around the conjugated particles, there will be a lot of dangling bonds, leading to great unsaturation. So it is easy to be combined with other atoms to form a stable state. Thus nano-material particles have great surface energy.

\section{The Production Process of Nano-concrete}

Due to the special natures of nano-materials, the dispersion problem is very important when nanomaterials are incorporated into the concrete. Maohua Zhang ${ }^{[1]}$, who combined the characteristics of 
nano-materials proposed that principle should be followed in the mixing of Nano concrete. (1) Due to the large surface of nano-materials, it can not allowed to be mixed with the aggregate in advance. So that it can avoid excessive nano-materials being adsorbed on aggregate and affecting the nanomaterials in cement dispersion. (2) Nano-materials can not be directly mixed with cement because nano-materials will be aggregated with cement when it is mixed with aggregates, thereby affecting the dispersion of nano-materials. (3) The use of dispersants can prevent a lot of nano-materials to be left in the container. (4) Nano-materials have a direct relationship with the speed of agitation and little to do with mixing time, so the best way is to use high-speed agitation. (5) Stir the nanomaterials, water, additives first, then put it into cement mixing material. It is possible to effectively improve the flow of concrete. (6) It is best to shock in high-frequency vibration units.

In the process of making nano-concrete, Fuzhou University Professor Tao $\mathrm{Ji}^{[2]}$ mixes part of water and all high efficiency water reducing agent first, then adds the nano-silica to the mixture and stirs. Although the nano-silica is insoluble in water, efficiency water reducing agent has a strong dispersing effect, and therefore nano-silica can be substantially uniform dispersed in the mixture. Then, put the cement and aggregate into compulsory mixer and adding some mixture, stirring 30s. Finally, pour the remaining mixture into compulsory mixer slowly and stir 60 s. This ensures the nano-material is dispersed in the concrete preferably.

\section{Nano-materials Research Status in Concrete}

The size of general cement particle is in the range of $7 \mu \mathrm{m} \sim 200 \mu \mathrm{m}$, but about $70 \%$ of its hydrates calcium silicate hydrate gel (C-S-H gel) is the size of the order of magnitude in the nanometer level. So hardened cement paste is actually condensed into calcium silicate hydrate primary nanomaterials, but it is very rough on the microstructure.

\section{The Pozzolanic Activity of Nano-materials}

Adding nano-materials (Nano-SiO $2, \mathrm{Nano}-\mathrm{CaCO}_{3}$, etc.) to the cement can not only change the flow of cement-based materials, but also can alter the microstructure of concrete, but the premise is appropriate dosage. In ordinary concrete, the internal concrete will precipitate calcium hydroxide on the age of one day. In $7 \mathrm{~d}$ age period, there will appear plenty of calcium hydroxide precipitates. These calcium hydroxide will appear in large numbers on the grout and the aggregate interface. These calcium hydroxide crystals have bad effects on the enhancing of the concrete strength. Nanomaterials (Nano- $\mathrm{SiO}_{2}, \mathrm{Nano}-\mathrm{CaCO}_{3}$, etc.) have a strong pozzolanic activity. It can promote the hydration of the cement slurry, generating dense calcium silicate hydrate (C-S-H). Network structure of C-S-H gel can significantly improve structural defects, the bonding properties of the structure as well as the structure and the paste between the gel and the aggregates. In addition, because the magnitude of nano-materials is in nano-meter level, it can effectively fill the tiny pores in the concrete, improve the internal pore structure, transform the harmful pores into harmless or less pores, reduce porosity, thus enhance the strength of concrete, toughness, resistance to ion permeability and durability and other properties.

Qing $\mathrm{Ye}^{[3]}$ compared pozzolanic activity between silicon powder and nano-silica and found that CSH gel could be easily detected in silicon powder slurry at the age of $28 \mathrm{~d}$ and $64 \mathrm{~d}$, while we could detect that there appears $\mathrm{CSH}$ gel at the age of $12 \mathrm{~h}$ and $1 \mathrm{~d}$ in nano-silica slurry. It is visible that the rate of producing calcium silicate hydrate slurry by mixing nano-silica and $\mathrm{Ca}(\mathrm{OH})_{2}$ is much bigger than the rate of calcium silicate hydrate slurry mixed by silicon powder and $\mathrm{Ca}(\mathrm{OH})_{2}$. So the pozzolanic activity of nanometer silica is much stronger than the silicon powder. At the same time it also affirms that the nano-materials have better results in terms of improving the performance of concrete. 


\section{Effect of Nano-materials on Concrete Strength}

Nano-materials (such as nano-silica, etc.) have good pozzolanic activity. In appropriate dosage range, it can improve the performance of concrete strength, toughness and the like. Zhejiang University Professor Qing Ye results ${ }^{[4]}$ showed that when the content of nano-silica is range from $1 \%$ to $3 \%$ (relative to the cement mass), it can significantly improve the mechanical properties of concrete. When the content of nano-silica is $2 \%$, the experimental results show that the strength of cement paste is increased by about $50 \%$ at the age for $7 \mathrm{~d}$ and $28 \mathrm{~d}$.

Haiyan Zhang, Yongjun $\mathrm{Wu}^{[5]}$ and others in the nano-concrete experimental study found that the strength of concrete show obvious increasing after mixing the concrete with nano-materials in a suitable water-cement ratio and a suitable dosage range of nano-materials. After analyzing the nanosilica concrete experimental data, they found that adding suitable dosage range of nano-silica to the convert with different water-cement ratio could increase the strength of concrete. When the watercement ratio is 0.3 , nano-silica content is $3.0 \%$, the increase of concrete strength is the most obvious. Compared with ordinary concrete, its strength increased by $11.3 \%$. But when the water-cement ratio continues to increase, the increase in concrete strength is less obvious. For this phenomenon, some researchers believe that it is because if the water-cement ratio increases, there will be a lot of water adsorbed on the surface of the nano-silica, and therefore the distribution of water to the cement hydration will be reduced. When the concrete interior humidity is reduced to a critical point, the hydration of the concrete will be reduced, or even stopped. Therefore, in this case, the strength of concrete would not be significantly improved.

In the study of nano calcium carbonate, Guhua $\mathrm{Li}$, Bo Gao ${ }^{[6]}$ believes that the nano calcium carbonate content is not the more the better. The best dosage is about $1.0 \%$. When the nano calcium carbonate content is at $1.0 \%$, the strength of concrete at $7 \mathrm{~d}, 28 \mathrm{~d}, 56 \mathrm{~d}$ increases by $33.3 \%, 7.7 \%$, $3.2 \%$. After XRD analysis, they get the initial judgment that there generates calcium carbonate hydrate, which is one of the important causes of nano calcium carbonate to improve the strength of concrete.

\section{The Effect of Nano-materials on Concrete Durability}

Guhua $\mathrm{Li}^{[7]}$ found that the resistance to corrosion coefficients of sulfate corrosion resistance of concrete in a month's corrosion experiments with the nano-silica content of $1.0 \%$, $2.0 \%$ were $0.87,0.92$. The corrosion resistance of concrete benchmark's coefficient was 0.84 . It showed that concrete corrosion incorporation of nano-materials in short period didn't have much improvement. After three months of sulfate corrosion, cracks appeared in the reference concrete's surface. The specimen was serious corrosion. The surface becomes crisp, and off phenomenon. The corrosion coefficient of is 0.23 . The corrosion resistance of concrete with incorporation of nano-silica $1.0 \%$ can be improved by $9.5 \%$. When add $2.0 \%$ of the incorporation of nano-silica, the corrosion resistance of concrete can be improved by $22.0 \%$. When the incorporation of nano-silica is $3.0 \%$, the corrosion coefficient increases to 0.82 . He believes that concrete is divided into two periods in the corrosion process by sulfates. In the early days, new generation of concrete salt crystals fill the pores in concrete, so the concrete is denser. And the strength of concrete at early age will be increased. Late, as the increasing of product inside the concrete, the internal forces will also continue to rise, and then it expanded. Finally the hole damage occurred leading to cracks and damage.

Yingji $\mathrm{Du}^{[8]}$ and others in the study of nano-concrete impermeability and frost experiments found that when the pressure was $1.20 \mathrm{MPa}$, the seepage height of ordinary concrete was $5.1 \mathrm{~cm}$, the seepage height of concrete with nano-materials was $1.7 \mathrm{~cm}$. He believes that with the addition of nano-materials, concrete internal holes (including large holes and pores) are a large number of reduction, porosity is reduced, the degree of compaction of concrete is also improved, and therefore the impermeability of concrete will be improved. Compared with the ordinary concrete, penetration resistance of concrete with nano-materials can increase by more than $30 \%$. In frost resistance experiments, the nano-concrete also has a good performance. Nano-materials can effectively 
plugging holes in size of $150 \mathrm{~nm}$ or less, reducing the number of water in the pores in concrete. After summarizing the experimental data, he find that compared with ordinary concrete, the frost resistance of concrete with nano-materials can be increased by more than $50 \%$.

Permeability is an important indicator of the evaluation of the concrete durability, to reduce the permeability of concrete can effectively prevent the infiltration from corrosive particles. As for the reinforced concrete, it is possible to prevent corrosion by reinforcing harmful particles through the protective layer to improve the concrete safety performance and service life. It is a good way to use resources more effectively and efficiently. Guhua $\mathrm{Li}^{[9]}$ find that adding suitable amount of nano calcium carbonate, the early resistance to chloride ion permeability of concrete has been significantly improved, while at a later stage, this good effect will be reduced. But adding excessive doped nano calcium carbonate, anti-permeability of the concrete will be reduced. The nano-silica can not only enhance early penetration resistance of concrete, but also can improve the performance of the late anti-infiltration. Incorporation of 3.0\% of nano-silica in concrete, when the age reaches $180 \mathrm{~d}$, the chloride ions through the concrete reduced by $31 \%$. When the content of nano-silica increases, the early penetration resistance of concrete increases. But later, it goes to the opposite with the previous.

\section{Conclusion}

Nanometer concrete in improving the performance of ordinary concrete (including mechanical properties, durability, etc.) has initially been affirmed, but its specific mechanism of action is not yet in place. The role played by them in concrete does not be mastered clearly and unambiguously. The further research and experimentation is needed.

At present, because of the production process of nano-materials and other reasons, its costs are still relatively high. It is not suitable for us to use in actual construction. Therefore, it is necessary to study and improve the production process to achieve lower costs. Only the costs are reduced, it is possible for it to enter the market, to enter the construction industry.

\section{Fund}

1 In Liaoning province innovation teams in Higher Education Project (Project Name: environmental geotechnical engineering; project number: LT2012021); 2 In Liaoning province university student Supporting of Innovation and Entrepreneurship Training Plan Project(Project Name: Experimental Study of new Nano-modified cement concrete performance; project number: 201411035000029)

\section{References}

[1] Zhang Maohua. The whole life of concrete pavement performance nanometer [D] Harbin: Harbin Institute of Technology, 2007.

[2] Ji Tao, Yuzhou Huang. Probe of Physical and Mechanical Properties of Nano-concrete, [J]. Concrete, 2003.161 (3).

[3] Ye Qing. Comparison of Pozzolanic Activity between Nano-SiO2 and Silica Fume [J]. Concrete, 2001.3

[4] Ye Qing. The Research and Development of Nano-composite Cement Structural Materials [J]. New Building Materials, 2001 (11).

[5] Zhang Haiyan, Wu Yongjun. Effect of Nano-SiO2 on the Mechanical Properties of High Performance Concrete [J]. New Building Materials, 2012.39 (7).

[6] Li GuHua, Gao Bo. The Impact of Nanometer Powders Si02and CaC03 on the Performance of Concrete [J]. Railway Society, 2006.28 (a).

[7] Li Guhua. Impact of Nano-materials on Durability of Concrete [D]. Southwest Jiaotong University, 2006.07.01. 
[8] Du Yingji, Han Sujian, Yao Yufang, Li Yuanting. The Improvement of Nano Powders on Impermeability and Frost Resistance of Concrete [J]. Northwest Agriculture and Forestry University (Natural Science), 2004.32 (7). 